\section{(6) OPEN ACCESS}

- Additional material is published online only. To view please visit the journal online (http://dx.doi.org/10.1136/ jclinpath-2015-202900).

'Department of Pathology, Fudan University Shanghai Cancer Center, Shanghai, China

2Department of Oncology, Shanghai Medical College, Fudan University, Shanghai, China

\section{Correspondence to} Professor Chao-Fu Wang, Departments of Pathology and Oncology, Fudan University Shanghai Cancer Center, Shanghai Medical College, Fudan University, No. 270 Dong An Road, Shanghai 200032, China; wangchaofu@126.com

YW, TW and P-PZ contributed equally.

Received 28 January 2015 Revised 21 April 2015 Accepted 25 June 2015 Published Online First 22 July 2015

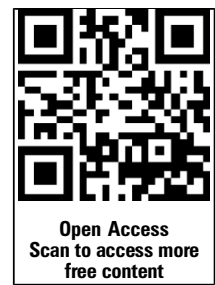

CrossMark

To cite: Wu Y, Wang T, Zhang P-P, et al. J Clin Pathol 2015;68:1020-1025.

\title{
Extraneural hemangioblastoma of the kidney: the challenge for clinicopathological diagnosis
}

\author{
Yong $\mathrm{Wu}_{1}{ }^{1,2}$ Tao Wang, ${ }^{1,2}$ Pei-Pei Zhang, ${ }^{1,2}$ Xiaoqun Yang, ${ }^{1,2}$ Jian Wang, ${ }^{1,2}$ \\ Chao-Fu Wang ${ }^{1,2}$
}

\section{ABSTRACT}

Background Hemangioblastoma is a benign cerebellar tumour which may occur as a sporadic entity or in association with von Hippel-Lindau (VHL) disease in approximately $25 \%$ of cases. Renal hemangioblastoma $(\mathrm{RH})$ is an extremely rare and newly recognised tumour. Here, we describe five cases of $\mathrm{RH}$, one discovered by $\mathrm{CT}$ in an accident and the other four detected during routine examinations.

Methods Five cases of renal hemangioblastoma retrieved from the Department of Pathology, Fudan University Shanghai Cancer Center were studied and the literatures were reviewed. Immunohistochemistry was used to differentiate and confirm this tumour.

Results Pathological examination following tumour resection revealed $\mathrm{RH}$ in all cases, the first patient was also diagnosed with renal cell carcinoma (RCC), suggesting the possibility of VHL syndrome, but PCR sequencing analysis of the VHL gene confirmed no mutation in any of the three exons, implying sporadic disease .Histologically, the tumours were circumscribed, composed of sheets of oval or polygonal cells and a prominent vascular network. Tumour cells had pleomorphic nuclei, but mitotic figures were rare. The diagnosis of hemangioblastoma was confirmed by immunohistochemistry.

Conclusions RH is very rare and is challenging to differentially diagnose. Distinguishing RCC and RH is difficult and each has a different prognosis, so differentiating between them is essential for avoiding over-diagnosis and unnecessary treatment.

\section{INTRODUCTION}

Hemangioblastoma is a benign tumour of uncertain histogenesis characterised by the presence of stromal cells and a rich vascular component. ${ }^{1}$ Most cases, which generally arise from the central nervous system (CNS), are sporadic, while approximately $25 \%$ are associated with von Hippel-Lindau (VHL) disease, an autosomal dominant disorder associated with germline mutations in the VHL tumour suppressor gene located on the short arm of chromosome $3 .^{23}$

Hemangioblastoma has also been reported outside the CNS, for example in the peripheral nerves, ${ }^{4-6}$ soft tissue, ${ }^{78}$ liver, ${ }^{9} 10$ lung, ${ }^{9}$ pancreas, ${ }^{11}$ kidney, ${ }^{11-15}$ retroperitoneum, ${ }^{16}$ urinary bladder, ${ }^{11}$ popliteal fossa ${ }^{7} 8$ and nasal skin. ${ }^{6}$ In this study, we describe five renal hemangioblastomas (RHs), all of which had features very similar to those of other malignancies such as renal cell carcinoma (RCC). We also describe the pathological and immunohistochemical features of this rare disease.

\section{MATERIALS AND METHODS}

All cases were sent to one of the authors for consultation. H\&E-stained sections (4 $\mathrm{mm}$ thickness) were re-examined to evaluate the tumour's histological features and immunohistochemistry was performed with an avidin-biotin-complex immunoperoxidase technique. Antibody details are given table 1 . Appropriate positive and negative controls were used throughout.

Clinical demographics and follow-up data were obtained from medical records and the referring physicians. The institutional review board for human studies of Fudan University Shanghai Cancer Center approved this retrospective study. Patient records or information were anonymised and de-identified prior to analysis. Written informed consent was provided by participants or their next-of-kin for their clinical records to be used in this study.

\section{RESULTS}

\section{Clinical characteristics of five cases}

Case 1

A 30-year-old man was admitted to the hospital for examination after an accident and a CT scan revealed a mass with a different density within a right kidney lesion. The malignancy was subsequently imaged by MRI. Surgical resection of the tumour revealed a collision tumour comprising RCC and extraneural hemangioblastoma, among which RCC accounts for less than $5 \%$ of the total tumour volume. The mass involved the renal cortex and medulla and measured $3.2 \times 2.5 \times 1.4 \mathrm{~cm}$. VHL disease was initially suspected. However, PCR sequencing analysis of the $V H L$ gene confirmed no mutation in any of the three exons, suggesting sporadic disease. The patient died before the next follow-up.

\section{Case 2}

A CT scan during a routine examination revealed that a 57-year-old woman had a mass in her right kidney. A radical nephrectomy was performed as a malignant tumour was suspected. H\&E slides confirmed the diagnosis of RCC. No surgery or chemotherapy was carried out. The patient is currently alive with no evidence of recurrent tumour or the development of other tumours.

\section{Case 3}

The patient was a 48-year-old man with no hereditary diseases. When he visited the hospital for evaluation of organ function, abdominal sonography revealed a tumour mass in the right kidney. A CT scan confirmed a $2.3 \mathrm{~cm}$ mass with heterogeneous density in the lower portion of the right kidney. As RCC was suspected, the 
Table 1 Panel of antibodies used in this study

\begin{tabular}{llll}
\hline Antigen & Clone & Dilution & Source \\
\hline S100 & Polyclonal & $1: 2000$ & $\begin{array}{l}\text { Dako, Carpinteria, } \\
\text { California, USA }\end{array}$ \\
Vimetin & V9 & $1: 200$ & Dako \\
EMA & E29 & $1: 500$ & Dako \\
HMB-45 & HMB-45 & $1: 50$ & Dako \\
CD34 & QBEnd10 & $1: 20$ & BioGenex, San Ramon, \\
CD31 & JC/70A & $1: 50$ & California, USA \\
PAX8 & Polyclonal & $1: 800$ & Dako \\
AE1/AE3 & AE1/AE3 & $1: 100$ & Chicago, Illinois, USA \\
Inhibin & R1 & $1: 50$ & Dako \\
NSE & BBS/NC/VI-H14 & $1: 200$ & Dako \\
CK7 & OV-TL 12/30 & $1: 50$ & Dako \\
CK8 & CAM5.2 & $1: 10$ & Dako \\
& & & Becton Dickinson, San Jose, \\
CK & AE1/AE3 & $1: 100$ & California, USA \\
\hline NSE, neuron-specific enolase. & & Dako \\
\hline
\end{tabular}

patient underwent a right radical nephrectomy. There was no evidence of tumour recurrence 42 months after the surgery. Follow-up magnetic resonance brain imaging did not any reveal tumours.

\section{Case 4}

A 25-year-old man was confirmed to have a left renal tumour during a routine examination, and was admitted to hospital for urological examination. The patient was asymptomatic with a normal appetite, no abdominal pain, no weight changes, and no family history of renal disease. CT imaging revealed a $3.6 \mathrm{~cm}$ well-defined, round, heterogeneously enhancing mass in the left kidney. Nephrectomy was performed and a $4.1 \mathrm{~cm}$ encapsulated tumour removed. Follow-up at 27 months confirmed no tumour recurrence or metastasis.

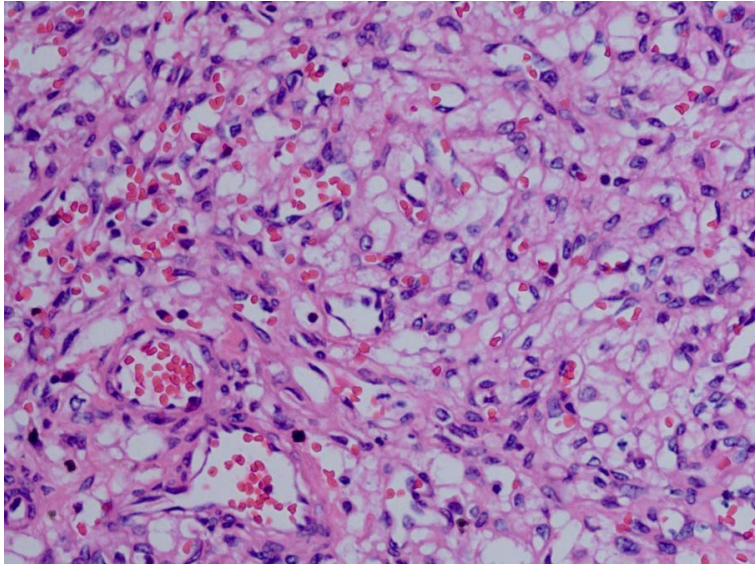

Figure 2 Blood vessels often had ectasia or pericytomatous configurations.

Case 5

A previously healthy 36-year-old woman presented with a tumour on the left kidney during a routine examination. The tumour was an unencapsulated but sharply circumscribed nodule. Clear cell RCC (CCRCC) was diagnosed. Immunohistochemistry of a tissue sample suggested a diagnosis of $\mathrm{RH}$. The patient was alive 3 months later.

\section{Microscopic features}

Microscopically, most tumours were well circumscribed and well demarcated from the surrounding renal parenchyma; only one tumour had areas of poorly marginated growth. The RHs all consisted of sheets of oval or polygonal cells traversed by a prominent, arborising vascular network (figure 1A, B). The tumours also included small microvacuolated cells with palely eosinophilic or clear cytoplasm (figure 1C, D). These microvacuolated cells often mimicked RCC. All tumours had a complex capillary network and blood vessels, which were thin-walled and lined with flat to plump endothelial cells, often had ectasia or pericytomatous configurations (figure 2). Case 1 had a focally marked nuclear
Figure 1 Histological features. (A) Most tumours were solid and were traversed by arborising thin-walled blood vessels. (B) Tumours were composed of plump spindle cells with palely eosinophilic cytoplasm. (C) The microvacuolated cells often mimicked lipoblasts or renal cell carcinoma and were mildly atypical. (D) Abundant anastomosing thin blood vessels surrounding bland-appearing stromal cells. Under a high-power field, scattered tumour cells have highly pleomorphic nuclei.
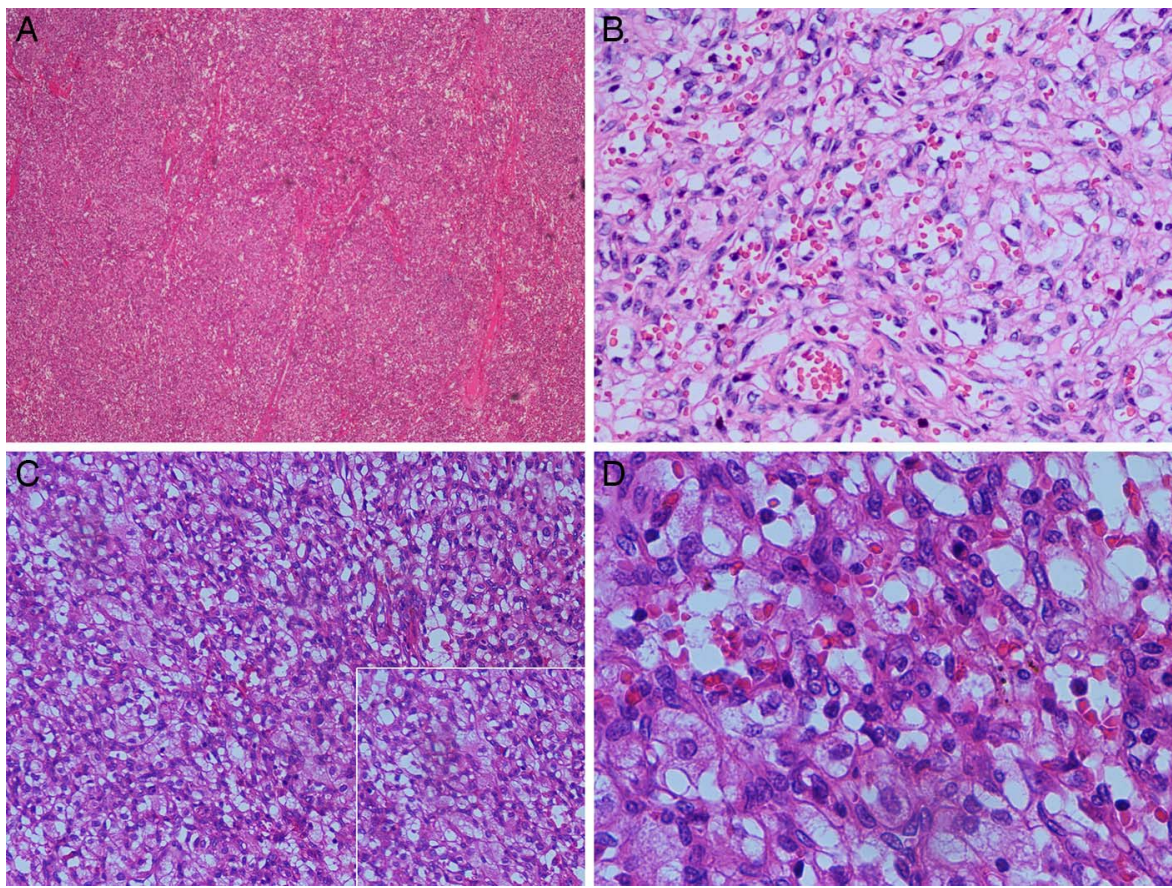
Figure 3 In most cases, tumour cells expressed inhibin (A) and vimentin (B).
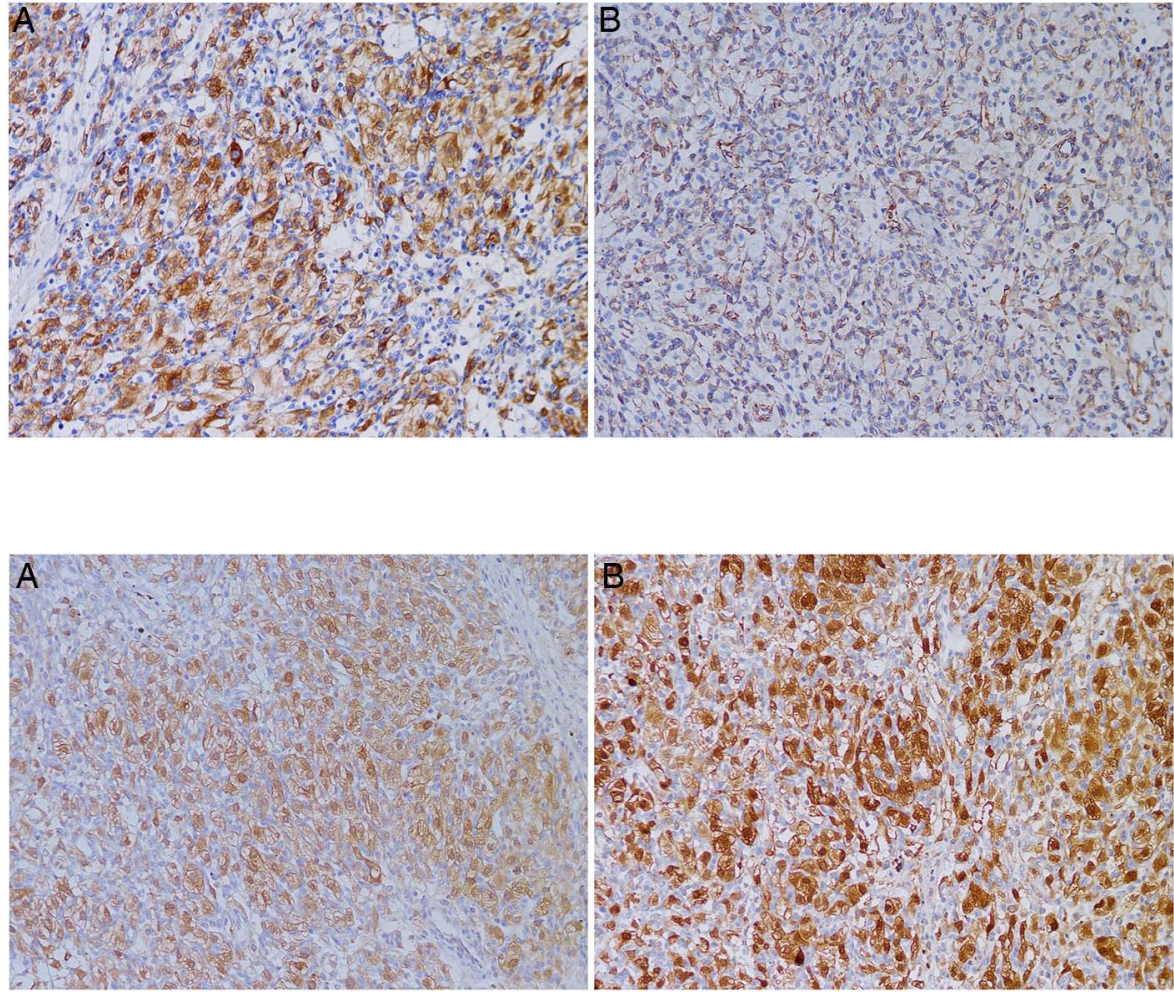

Figure 4 Tumour cells were positive for neuron-specific enolase $(A)$ and S100 protein (B). pleomorphism; nuclear pleomorphism of the other cases were generally difficult to detect. There were a very few mitotic figures in one case and none at all in the other cases. In all cases, there were minimal areas of stromal hyalinisation. Necrosis or lymphovascular invasion was not identified in any case.

\section{Immunohistochemical findings}

Most cases had similar immunohistochemical profiles. Tumour cells expressed inhibin (3/5) and vimentin (3/5) in 60\% of cases (figure 3A, B). Neuron-specific enolase (NSE) and S100 protein were expressed in all cases (figure 4A, B). EMA was focally positive in stromal cells in half of the cases (figure 5). Four of the tumours were negative for CK, CK7, CK8, PAX8, AE1/AE3 and HMB45 (figure $6 \mathrm{~A}-\mathrm{F}$ ). CD31 or CD34 stains highlighted the capillary network but not tumour cells (figure 7). Interestingly, one case had markers for both hemangioblastoma and CCRCC, suggesting the

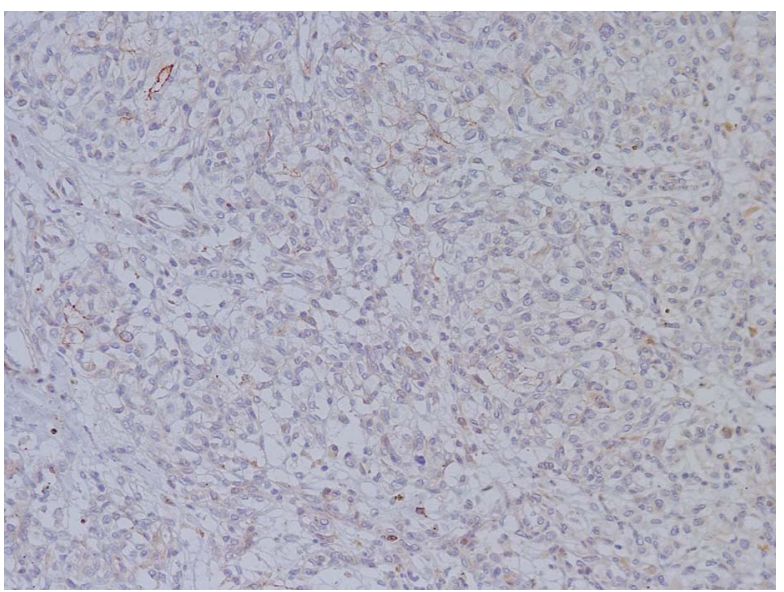

Figure 5 EMA was focally positive in stromal cells in some cases. possibility of VHL syndrome. Immunohistochemistry data are summarised in table 2.

\section{DISCUSSION}

Hemangioblastoma is a benign tumour of uncertain histogenesis that generally occurs in a relatively restricted area of the CNS. ${ }^{17}$ Extraneural cases are seldom seen and usually accompany VHL disease. ${ }^{13}$ This report describing two female and three male patients (median age 39.2 years; range 25-57 years) with $\mathrm{RH}$ adds to the total number of published cases (table 3 ). In contrast to CNS hemangioblastoma which is predominantly found in males, renal tumours affect both male and female patients. ${ }^{1} 18$ CNS hemangioblastoma is found predominantly in the third to the fifth decades of life. However, patients in our series were younger than those in previous reports, but this may not be significant as our sample size was small.

VHL disease is an autosomal dominant hereditary disorder characterised by retinal and CNS hemangioblastomas, pheochromocytoma and CCRCC. ${ }^{19-21}$ Mutations in the VHL gene lead to the development of several benign or malignant tumours, and cysts in many organ systems. The VHL gene which is located on chromosome $3 \mathrm{p} 25$, encodes for a 213 amino acid tumour suppressor protein that plays a key role in the regulation of the hypoxia response pathway. ${ }^{2}{ }^{22}$ Aberrations in VHL function, either through mutation or other mechanisms, lead to the accumulation of hypoxia-inducible factor, which then transcriptionally upregulates a sequence of hypoxia responsive genes, including epidermal growth factor, vascular endothelial growth factor, platelet-derived growth factor and other pro-angiogenic factors, resulting in tumour formation. $^{23}$ It is currently recommended that all patients with CNS hemangioblastoma undergo testing for VHL germline mutations. According to the WHO Blue Book, the clinical diagnosis of VHL disease is based on the presence of hemangioblastoma in the CNS or retina, the presence of one of the 
Figure 6 Most tumours except case 1 were negative for CK (A), CK7 (B), CK8 (C), PAX8 (D), AE1/AE3 (E) and HMB45 (F).
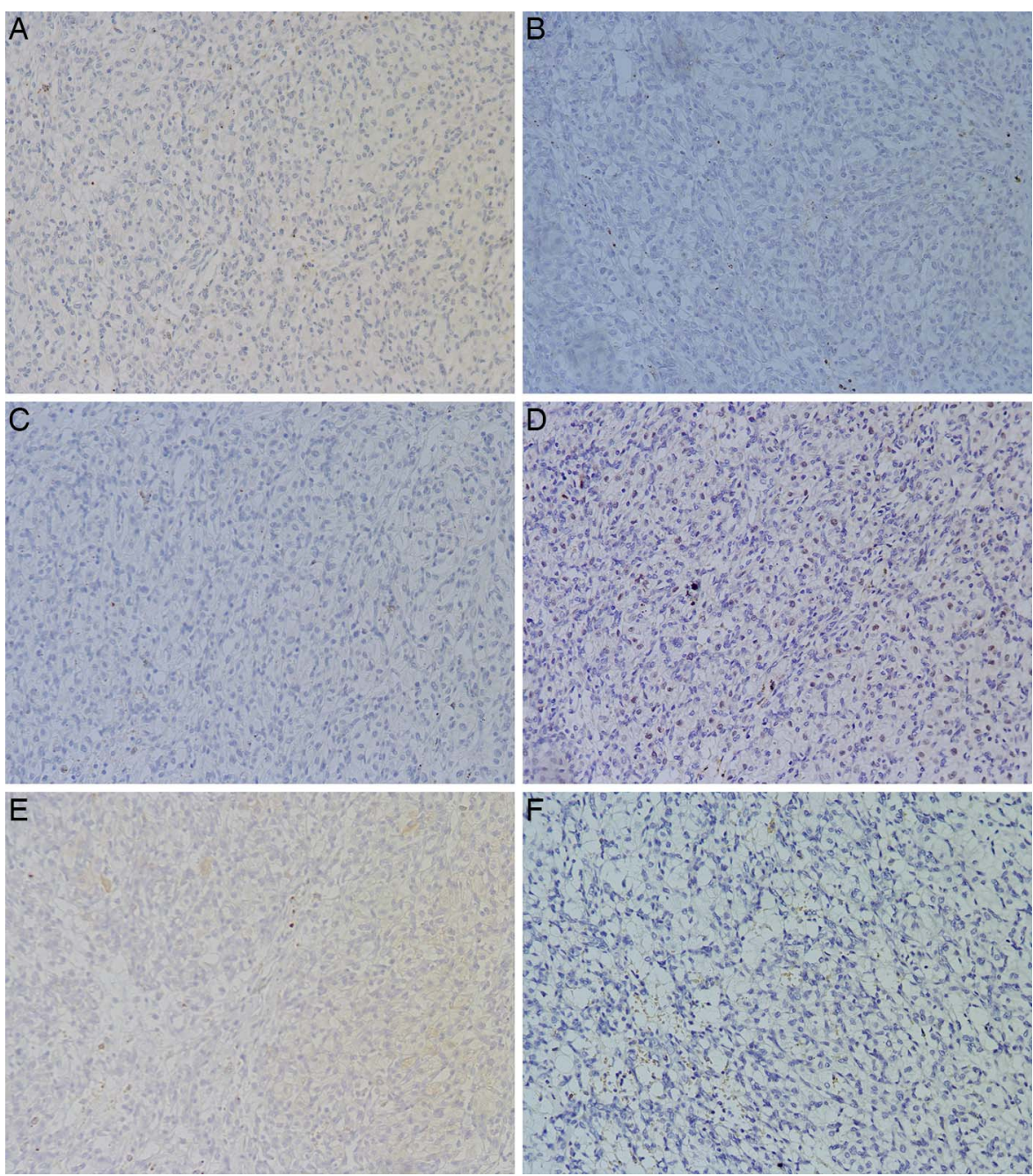

typical VHL-associated tumours, or a previous family history. ${ }^{1}$ In the present study, one case which expressed markers of hemangioblastoma and CCRCC was subjected to Sanger DNA sequencing. Each exon was identified and confirmed by both forward and reverse directional analyses but no mutation was detected. None of the other patients met any of these criteria, and are considered to have sporadic disease.

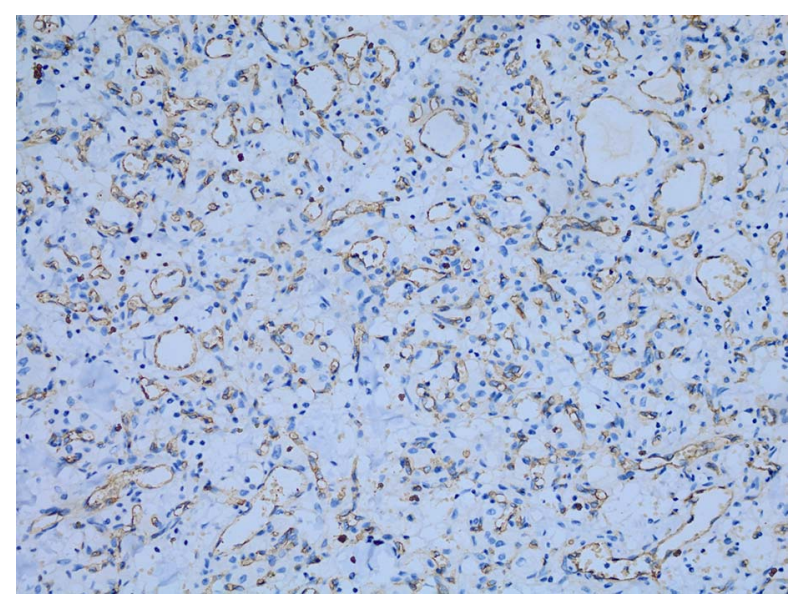

Figure 7 CD34 stains highlighted the capillary network but not the tumour cells.
The tumours in these five cases were morphologically similar to lesions arising in the CNS, which are also composed of sheets of oval or polygonal cells with palely eosinophilic and

Table 2 Results of immunohistochemical staining in renal hemangioblastoma $(\mathrm{RH})$

\begin{tabular}{lllllll}
\hline & \multicolumn{7}{c}{ Case 1 } & & & & \\
\cline { 2 - 5 } & RH & RCC & Case 2 & Case 3 & Case 4 & Case 5 \\
\hline Vimentin & + & + & + & - & - & + \\
S100 & + & - & + & + & + & + \\
Inhibin & + & - & - & + & - & + \\
NSE & + & + l- & N/A & + & + & + \\
EMA & + & + & - & - & N/A & + \\
CK7 & - & + & - & - & - & - \\
CK8 & - & + & - & - & - & - \\
CK & N/A & N/A & - & - & N/A & - \\
CD10 & - & + & - & - & - & + \\
AE1/AE3 & - & + & - & - & - & - \\
HMB45 & N/A & N/A & N/A & - & - & - \\
CD31 & N/A & N/A & + & N/A & N/A & + \\
CD34 & + & - & + & + & + & + \\
PAX8 & - & + & - & - & - & - \\
\hline NA, stain not performed; NSE, neuron-specific enolase; RCC, renal cell carcinoma; \\
,+ positive; -, negative; +l-, variable.
\end{tabular}


Table 3 Reported cases of renal hemangioblastoma

\begin{tabular}{|c|c|c|c|c|}
\hline References & Age & Gender & Presence of VHL & Outcome \\
\hline Nonaka et $a l^{32}$ & 71 & $\mathrm{~F}$ & Not stated & No recurrence 9 years after nephrectomy \\
\hline Ip et $\left.a\right|^{13}$ & 58 & M & No & No recurrence 2 years after nephrectomy \\
\hline Ip et $\left.a\right|^{13}$ & 55 & $\mathrm{~F}$ & No & Well after nephrectomy \\
\hline Wang et $a l^{15}$ & 29 & M & No & No recurrence 20 months after nephrectomy \\
\hline Verine et $a l^{33}$ & 64 & M & No & No recurrence 1 year after nephrectomy \\
\hline Wang et $a l^{14}$ & 61 & M & No & No recurrence 1 year after nephrectomy \\
\hline Liu et $a l^{36}$ & 36 & $\mathrm{~F}$ & No & No recurrence 1 year after nephrectomy \\
\hline Current case 1 & 30 & M & Suggestive of VHL & Died after nephrectomy \\
\hline Current case 2 & 48 & M & No & No recurrence 3.5 years after nephrectomy \\
\hline Current case 3 & 25 & M & No & No recurrence 27 months after nephrectomy \\
\hline Current case 4 & 36 & $\mathrm{~F}$ & No & Well after nephrectomy \\
\hline Current case 5 & 57 & $\mathrm{~F}$ & No & No recurrence 5 months after nephrectomy \\
\hline
\end{tabular}

clear microvacuolated cytoplasm, separated by a delicate capillary network, and usually with interspersed larger thin-walled and thick-walled blood vessels.

Compared with CNS hemangioblastoma, the results of immunohistochemical staining reported by many authors do not suggest a specific line of differentiation for $\mathrm{RH}$ tumours. $^{16}$ 24-27 Markers currently used for these lesions include epithelial (cytokeratins), muscle (MSA, desmin, calponin), neural (S100 protein, neuron specific enolase) and other generic mesenchymal markers (vimentin); vimentin, S100 protein, NSE and inhibin are reported to express constantly in this tumour.

In the current study, S100 and NSE appeared to be the most sensitive markers and were expressed in all cases, as was inhibin. Vimentin expression was observed in $60 \%$ of cases. Of note, expression of inhibin was less in our cases than reported in the $\mathrm{RH}$ literature; sample limitations may explain this discrepancy. The fact that vimentin is strongly expressed in these tumours has led investigators to suggest they have an undifferentiated mesenchymal origin. ${ }^{4}$ Expression of GLUT1 in CNS hemangioblastoma has been reported to be helpful for differential diagnosis with metastatic RCC, as is the strong endothelial staining observed with most hemangioblastomas, unlike RCC, which does not show such a staining pattern. ${ }^{28}$ However, this finding is controversial, as some authors ${ }^{29}$ doubt the low specificity and tendency for high background staining. Recently, expression of brachyury has been described in cerebellar hemangioblastoma tumour cells. $^{30} 31$ Tirabosco's group ${ }^{30}$ reported nuclear expression of brachyury in stromal cells in all 14 CNS cases they examined. However, Doyle and Fletcher ${ }^{29}$ reported that brachyury was not expressed in extraneural tumours in their series.

$\mathrm{RH}$ is frequently misdiagnosed as RCC, and is likely to be under-recognised as it mimics many tumour types morphologically and is usually not considered in a differential diagnosis. ${ }^{32}$ Correctly diagnosing $\mathrm{RH}$ is challenging and important because sporadic RH does not require further treatment and the prognosis is much better than that of malignant RCC. ${ }^{14} 15$ The presence of pericytomatous growth patterns and intracytoplasmic lipid vacuoles strongly suggests hemangioblastoma, ${ }^{13} 33$ although both tumour types have similar morphological features, such as clear cytoplasm and a vascular network. ${ }^{34}$ Immunohistochemistry is useful for differentiating morphologically similar neoplasms. RCC is usually positive for cytokeratin (predominantly low molecular weight), PAX8 and
AE1/AE3. ${ }^{27} 34$ However, the immunohistochemistry data for cytokeratin, PAX8, and AE1/AE3 ruled out the possibility of RCC in our study. In contrast to hemangioblastoma, RCC is usually negative for inhibin, S100 and NSE. Other differential diagnoses include adrenal cortical carcinoma, epithelioid angiomyolipoma and paraganglioma, ${ }^{35}$ which can offer critical diagnostic information.

\section{Take home messages}

- Extraneural renal hemangioblastoma (RH) is a rare, newly recognised tumour with morphological features similar to its cerebellar counterpart.

- This report describes the morphological features and immunohistochemical characteristics of five cases of RH and summarises the findings.

- RH is frequently misdiagnosed, so immunohistochemistry is essential for differentiating RH from morphologically similar neoplasms, such as renal cell carcinoma.

Handling editor Cheok Soon Lee

Contributors YW and TW collected and analyse the data. XY help to analyse the data and examine the H\&E slides. YW and P-PZ are responsible for writing the paper. C-FW and JW are responsible for the entire experiment and correcting the manuscript.

Competing interests None declared.

Patient consent Obtained.

Ethics approval The institutional review board for human studies of Fudan University Shanghai Cancer Center approved this retrospective study.

Provenance and peer review Not commissioned; externally peer reviewed.

Open Access This is an Open Access article distributed in accordance with the terms of the Creative Commons Attribution (CC BY 4.0) license, which permits others to distribute, remix, adapt and build upon this work, for commercial use, provided the original work is properly cited. See: http://creativecommons.org/ licenses/by/4.0/

\section{REFERENCES}

1 Louis DN, Ohgaki H, Wiestler OD, et al. The 2007 WHO classification of tumours of the central nervous system. Acta Neuropathol 2007;114:97-109.

2 Latif F, Tory K, Gnarra J, et al. Identification of the von Hippel-Lindau disease tumor suppressor gene. Science 1993;260:1317-20. 
3 Lonser RR, Glenn GM, Walther M, et al. von Hippel-Lindau disease. Lancet 2003:361:2059-67.

4 Giannini C, Scheithauer BW, Hellbusch LC, et al. Peripheral nerve hemangioblastoma. Mod Pathol 1998:11:999-1004.

5 Rasulic L, Samardzic M, Bascarevic V, et al. A rare case of peripheral nerve hemangioblastoma-case report and literature review. Neurosurg Rev 2015:38:205-9.

6 Brodkey JA, Buchignani JA, O'Brien TF. Hemangioblastoma of the radial nerve: case report. Neurosurgery 1995;36:198-200; discussion 00-1.

7 Patton KT, Satcher RL Jr, Laskin WB. Capillary hemangioblastoma of soft tissue: report of a case and review of the literature. Hum Pathol 2005;36:1135-9.

8 Michal M, Vanecek T, Sima R, et al. Primary capillary hemangioblastoma of peripheral soft tissues. Am J Surg Pathol 2004;28:962-6.

9 McGrath FP, Gibney RG, Morris DC, et al. Case report: multiple hepatic and pulmonary haemangioblastomas - a new manifestation of von Hippel-Lindau disease. Clin Radiol 1992;45:37-9.

10 Rojiani AM, Owen DA, Berry K, et al. Hepatic hemangioblastoma. An unusual presentation in a patient with von Hippel-Lindau disease. Am J Surg Pathol 1991;15:81-6.

11 Bird AV, Mendelow H. Lindau's disease in a South African family a report on three further cases. Br J Surg 1959;47:173-6.

12 Christoferson LA, Gustafson MB, Petersen AG. Von Hippel-Lindau's disease. JAMA 1961;178:280-2.

13 Ip YT, Yuan JQ, Cheung $H$, et al. Sporadic hemangioblastoma of the kidney: an underrecognized pseudomalignant tumor? Am J Surg Pathol 2010;34:1695-700.

14 Wang Y, Wei C, Mou L, et al. Sporadic renal haemangioblastoma: case report and review of the literature. Oncol Lett 2013;5:360-2.

15 Wang CC, Wang SM, Liau JY. Sporadic hemangioblastoma of the kidney in a 29-year-old man. Int J Surg Pathol 2012;20:519-22.

16 Fanburg-Smith JC, Gyure KA, Michal M, et al. Retroperitoneal peripheral hemangioblastoma: a case report and review of the literature. Ann Diagn Pathol 2000:4:81-7.

17 Hussein MR. Central nervous system capillary haemangioblastoma: the pathologist's viewpoint. Int J Exp Pathol 2007;88:311-24.

18 Ironside JW, Moss TH, Louis DN, et al. Diagnostic pathology of nervous system tumours. Churchill Livingstone London, 2002

19 Cowey CL, Rathmell WK. VHL gene mutations in renal cell carcinoma: role as biomarker of disease outcome and drug efficacy. Curr Oncol Rep 2009;11:94-101.

20 Gnarra JR, Tory K, Weng Y, et al. Mutations of the VHL tumour suppressor gene in renal carcinoma. Nat Genet 1994;7:85-90.

21 Lee JY, Dong SM, Park WS, et al. Loss of heterozygosity and somatic mutations of the VHL tumor suppressor gene in sporadic cerebellar hemangioblastomas. Cancer Res 1998;58:504-8.
22 Iliopoulos 0 , Levy AP, Jiang $C$, et al. Negative regulation of hypoxia-inducible genes by the von Hippel Lindau protein. Proc Natl Acad Sci USA 1996;93:10595-9.

23 Wiesener MS, Munchenhagen PM, Berger I, et al. Constitutive activation of hypoxia-inducible genes related to overexpression of hypoxia-inducible factor-1 alpha in clear cell renal carcinomas. Cancer Res 2001;61:5215-22.

24 Frank TS, Trojanowski JQ, Roberts SA, et al. A detailed immunohistochemical analysis of cerebellar hemangioblastoma: an undifferentiated mesenchymal tumor. Mod Pathol 1989:2:638-51.

25 Ishizawa K, Komori T, Hirose T. Stromal cells in hemangioblastoma: neuroectodermal differentiation and morphological similarities to ependymoma. Pathol Int 2005;55:377-85.

26 Hoang MP, Amirkhan RH. Inhibin alpha distinguishes hemangioblastoma from clear cell renal cell carcinoma. Am J Surg Pathol 2003:27:1152-6.

27 Weinbreck N, Marie B, Bressenot A, et al. Immunohistochemical markers to distinguish between hemangioblastoma and metastatic clear-cell renal cell carcinoma in the brain: utility of aquaporin 1 combined with cytokeratin AE1/AE3 immunostaining. Am J Surg Pathol 2008;32:1051-9.

28 North PE, Mizeracki A, Mihm MC Jr, et al. GLUT1 immunoreaction patterns reliably distinguish hemangioblastoma from metastatic renal cell carcinoma. Clin Neuropathol 2000;19:131-7.

29 Doyle LA, Fletcher CD. Peripheral hemangioblastoma: clinicopathologic characterization in a series of 22 cases. Am J Surg Pathol 2014;38:119-27

30 Tirabosco R, Mangham DC, Rosenberg AE, et al. Brachyury expression in extra-axial skeletal and soft tissue chordomas: a marker that distinguishes chordoma from mixed tumor/myoepithelioma/parachordoma in soft tissue. Am J Surg Pathol 2008;32:572-80.

31 Barresi V, Vitarelli E, Branca $G$, et al. Expression of brachyury in hemangioblastoma potential use in differential diagnosis. Am J Surg Pathol 2012;36:1052-7.

32 Nonaka D, Rodriguez J, Rosai J. Extraneural hemangioblastoma: a report of 5 cases. Am J Surg Pathol 2007;31:1545-51.

33 Verine J, Sandid W, Miquel C, et al. Sporadic hemangioblastoma of the kidney: an underrecognized pseudomalignant tumor? Am J Surg Pathol 2011;35:623-4.

34 Amin MB, McKenney J, Tickoo S, et al. Diagnostic pathology: genitourinary. Altona MB, Canada: Amirsys, 2010

35 Lapinski JE, Chen L, Zhou M. Distinguishing clear cell renal cell carcinoma, retroperitoneal paraganglioma, and adrenal cortical lesions on limited biopsy material: utility of immunohistochemical markers. Appl Immunohistochem Mol Morphol 2010;18:414-21

36 Liu Y, Qiu XS, Wang EH. Sporadic hemangioblastoma of the kidney: a rare rena tumor. Diagn Pathol 2012;7:49. 